\title{
Fiber dissection and 3-tesla diffusion tensor tractography of the superior cerebellar peduncle in the human brain: emphasize on the cerebello-hypthalamic fibers
}

\author{
Safiye Çavdar ${ }^{1}$ (D) Ayşegül Esen Aydın ${ }^{2} \cdot$ Oktay Algin $^{3,4} \cdot$ Evren Aydoğmuş $^{5}$
}

Received: 24 September 2019 / Accepted: 9 November 2019 / Published online: 27 November 2019

○) Springer-Verlag GmbH Germany, part of Springer Nature 2019

\begin{abstract}
Experimental studies in various species using tract-tracing techniques showed clear evidence of the presence of cerebellohypothalamic projections. However, these connections were not clearly described in humans. In the present study we aimed to describe the direct cerebello-hypothalamic connections within the superior cerebellar peduncle (SCP) using fiber dissection techniques on cadaveric brains and diffusion tensor tractography (DTI) in healthy adults. Fiber dissection was performed in a stepwise manner from lateral to medial on 6 cerebral hemispheres. The gray matter was decorticate and fiber tracts were revealed. The SCP was exposed and the fibers were traced distally using wooden spatulas. The MRI examinations were performed in seven cases using 3-tesla 3T unit. The direct cerebello-hyothalamic pathways were exposed using high-spatialresolution DTI. The present study using both fiber dissection and DTI in adult human showed direct cerebello-hypothalamic fibers within the SCP. The SCP fibers course anterolateral to the cerebral aqueduct reaching the level of the red nucleus of the midbrain. The majority of the fibers crosses over and reached the contralateral diencephalic structures and some of these fibers terminated at the contralateral anterior hypothalamic area. Some of the uncrossed SCP fibers reached the ipsilateral diencephalic structures and terminated at the ipsilateral posterior hypothalamic area. We further reported the close relationship of the SCP with the MCP, lateral lemniscus, red nucleus and substantia nigra. In the DTI evaluations of the SCP we exposed unilateral left cerebello-hypothalamic fibers in five cases and bilateral cerebello-hypothalamic fibers in two cases. The present study demonstrates the direct cerebello-hypothalamic connections within the SCP for the first time using fiber dissection and DTI technique in the human brain. The detailed knowledge of the cerebello-hypothalamic fibers can outline the unexplained deficit that may occur during regional surgery.
\end{abstract}

Keywords Cerebello-hypthalamic · Fiber dissection · Diffusion tensor tractography · Human

\section{Introduction}

Safiye Çavdar

scavdar@ku.edu.tr

1 Department of Anatomy, School of Medicine, Koç University, Sarıyer, 34450 Istanbul, Turkey

2 Department of Neurosurgery, Bakırköy Ruh ve Sinir Hastanesi, Istanbul, Turkey

3 Radiology Department, City Hospital, Yıldırım Beyazıt University, Ankara, Turkey

4 National MR Research Center (UMRAM), Bilkent University, Ankara, Turkey

5 Department of Neurosurgery, Dr. Lütfi Kirdar Kartal Education and Research Hospital, Istanbul, Turkey
The role of cerebellum in controlling motor movements and motor learning has been well documented in literature (Wayner et al. 1991; Anderson et al. 1987). Electrophysiological and tract-tracing studies in various species showed vast connections of the cerebellum with cortical, subcortical and brainstem structures (Cohen and Yarom 2000; Cavdar et al. 2001, 2018a, b). Experimental studies using animals showed connections of the cerebellum with nonmotor areas of the central nervous system (Cavdar et al. 2001; Middleton and Strick 1997, 1998). Further, clinical studies have shown the involvement of cerebellum in cognitive functions such as attention, language, regulating fear and pleasure responses (Haines and Manto 2007) and autonomic functions such as controlling the blood pressure, respiratory rate, heart rate, 
bladder tone or GI motility (Ban et al. 1956; Haines et al. 1997; Zheng et al. 1982; Chida et al. 1986; Zanchetti and Zoccolini 1954).

The influence of the cerebellum on non-motor regulation is known to be through the cerebello-hypothalamic pathways. The reciprocal connections of the cerebellum with the hypothalamus has been documented in rat, cat (Cavdar et al. 2001, 2018; Haines and Dietrichs 1984, 1985; Dietrichs and Zheng 1984) and monkey (Dietrichs and Zheng 1984) using tract tracing and electrophysiology (Dietrichs and Zheng 1984; Dietrichs et al. 1985; Haines and Dietrichs 1984; Haines et al. 1990. The hypothalamo-cerebellar fibers arise from the lateral, posterior, dorsal hypothalamic area, the dorsomedial and ventromedial nuclei and paraventricular zone and terminate in all layers of the cerebellar cortex (Haines and Dietrichs 1984). Cerebello-hypothalamic fibers arise from all four deep cerebellar nuclei and terminate in the lateral, posterior and dorsal hypothalamic area and in the dorsomedial and paraventricular nuclei Cavdar et al. 2001, 2018a, b; Haines and Dietrichs 1984).

In our previous studies we have clearly demonstrated the cerebello-hypothlamic connections using tract tracing methods in the rat (Cavdar et al. 2001, 2018a, b). Further, the majority of these cerebellar connections were localized within the SCP (brachium conjunctivum). In the present study, we aimed to expose the direct cerebello-hypothalamic projections within the SCP using fiber dissection technique in human brain and diffusion tensor tractography (DTI) in healthy adults which has not been described in the literature. Furthermore, the course and the anatomical relations of the SCP will be defined.

\section{Materials and methods}

\section{Fiber dissection technique}

In the present study, six formalin-fixed brain hemispheres were used. The age of the cadavers ranged between 64 and 76 years-old (mean, 67 years). None had grossly visible abnormalities of the brain. The brains were keep in $10 \%$ formalin solution for 3 weeks then were frozen and stored at $-163{ }^{\circ} \mathrm{C}$ for 2 weeks before dissection (Klingler and Ludwig 1956). Prior to the fiber dissection the dura, arachnoid matter and the vessels were carefully removed from the specimens using a dissection microscope. All fibers dissection was performed under X6-X40 magnification using Zeiss Surgical Microscope (Carl Zeiss AG, Oberkochen, Germany). Handmade thin, wooden spatulas were used for dissection. The fiber bundles were peeled along the anatomic planes. All specimens were stored in $70 \%$ alcohol solution during entire study period. Three dimensional images were taken and stored for every stage of the dissections.

\section{Diffusion tensor imaging (DTI) tractography of the cerebello-hypothalamic fibers}

The MRI examinations of seven cases (five males and two females, age range 20-30), using a 3T unit (Trio with Tim; Siemens Healthcare AG, Erlangen, Germany) with 32-channel birdcage head coil. During MRI data acquisition, subjects were positioned with head in supine position in the MRI scanner. For anatomical comparisons, we also included high-resolution anatomical imaging using a 9-min T1-weighted axial magnetization-prepared rapid-acquisition gradient-echo sequence (repetition time $=2110 \mathrm{~ms}$, echo time $=2.63 \mathrm{~ms}$, flip angle $=8$, number of slices $=176$, field of view $=256 \times 256 \mathrm{~mm}^{2}$, voxel size $=0.5 \times 0.5 \times 1.0 \mathrm{~mm}^{3}$ ). Tractography data was obtained as mentioned below; DWI: TR $7750 \mathrm{~ms}$, TE: $89 \mathrm{~ms}$, FoV: $237 \mathrm{~mm}$, matrix: $128 \times 128$, slice thickness: $1.8 \mathrm{~mm}$, distance factor: $0 \%, 60$ slices, GRAPPA with factor 2, voxel size: $1.9 \times 1.9 \times 1.9 \mathrm{~mm}^{3}$, diffusion encoding gradients: $60, b$ values: $0-1000 \mathrm{~s} / \mathrm{mm}^{2}$, orientation: transverse, phase encoding direction: anterior-posterior, data acquisition time: about $9 \mathrm{~min}$. The measurement volume of DWI was aligned in parallel to the connecting line of the anterior and posterior commissure. Neuro 3D software was used for data analysis (Siemens Healthcare AG, Germany).

\section{Results}

\section{Fiber dissections}

Fiber dissections were continued from lateral towards the medial aspect of the hemispheres. The cortical gray matter on the frontal, parietal and temporal operculum was removed and the insular cortex and the arcuate fasciculus were visualized (Fig. 1). After the removal of the insular cortex the extreme capsule, external capsule, inferior fronto-occipital fasciculus (IFOF) and the claustrum was exposed (Fig. 2). The superior, middle and the inferior temporal gyri were removed and the inferior horn of the lateral ventricle and the choroid plexus within the ventricle were visualized (Fig. 2). Furthermore, the sagittal stratum and the arcuate fascicle were exposed (Fig. 2). In the next step, the lentiform nucleus (putamen and globus pallidus) and inferiorly located anterior commissure was exposed (Figs. 3, 4). Deep to the anterior aspect of the parahippocampus, pes hippocampi was exposed (Fig. 3). The parahippocampal gyrus was removed and the hippocampus forming the floor of the inferior horn of the lateral ventricle has been exposed (Fig. 4). By removing the anterior limb of the internal capsule the caudate nucleus, 


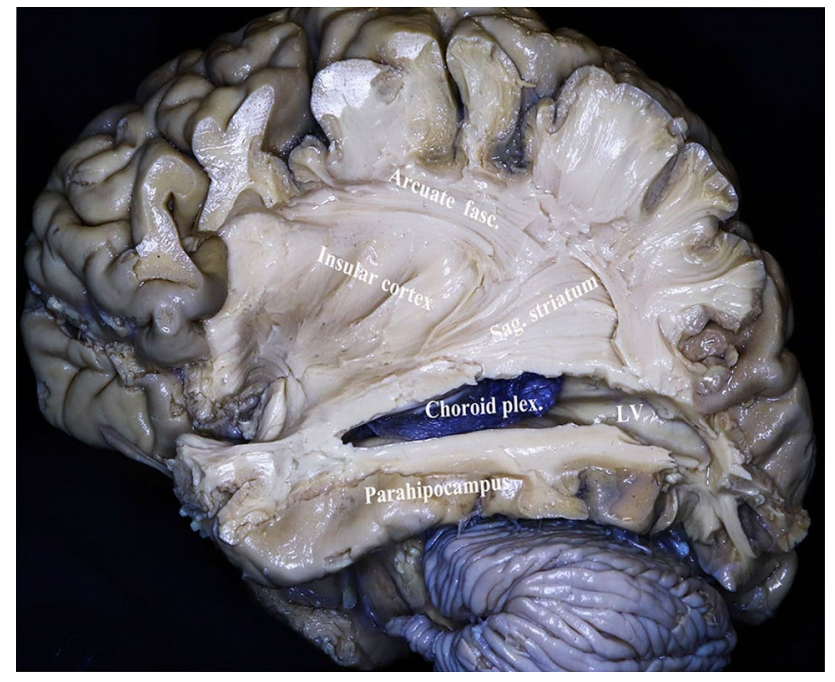

Fig. 1 The superior, middle and the inferior temporal gyri were removed and the inferior horn of the lateral ventricle and the choroid plexus within the ventricle were exposed. The cortical gray matter on the frontal, parietal and temporal operculum was removed and the insular cortex was visualized. The sagittal stratum and the arcuate fascicle was visualized

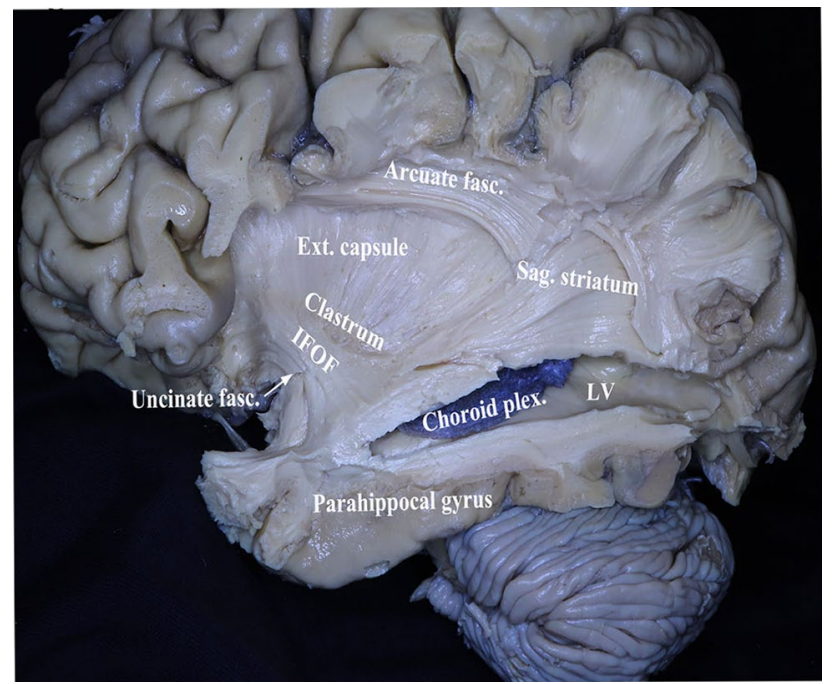

Fig. 2 The insular cortex was removed and clastrum, external capsule, inferior frontooccipital fasciculus (IFOF) and was uncinate fasciculus was exposed

and removing the posterior limb of the internal capsule the thalamus was exposed (Figs. 5, 6). The lower fibers of the internal capsule and the optic nerve were removed and the tail of the caudate nucleus was revealed (Fig. 6). Further continuing the fiber dissection from lateral to medial, removing the left thalamus the corticospinal fibers were exposed (Fig. 6). Then after removing the corticospinal fibers we reached the thalamus, hypothalamus, mammillary body of the opposite hemisphere (Fig. 7). Inferiorly

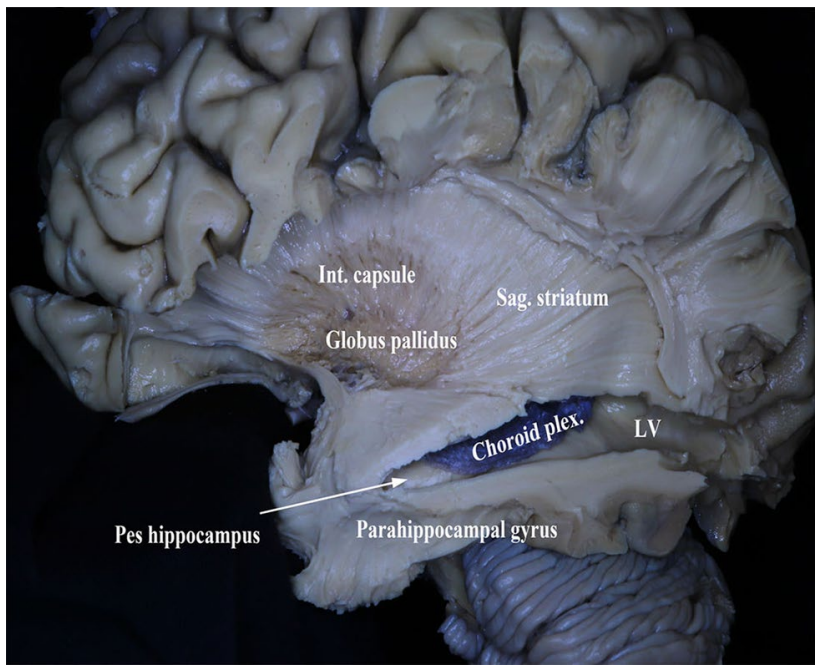

Fig. 3 The putamen has been removed and the globus pallidus and the internal capsule was revealed

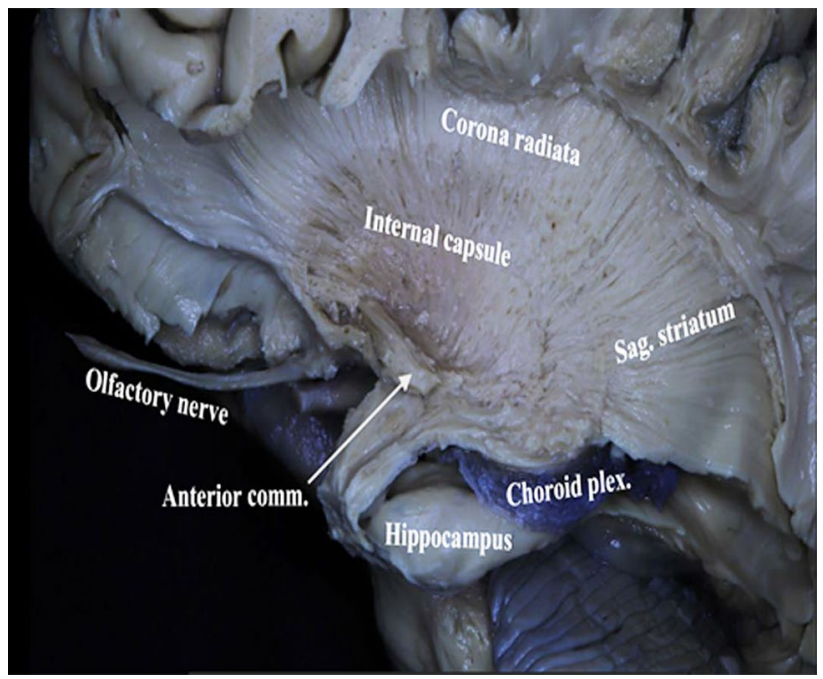

Fig. 4 Deep to the anterior aspect of the parahippocampus pes hippocampi was observed

the SCP was localized anterior and lateral to the MCP and ascended towards the midbrain (Fig. 7). The lateral lemniscus was localized anterior-inferior to the left SCP. Further, the tectal structures the superior and inferior colliculus was removed. The left SCP was traced until the tegmentum. The SCP was localized posterior and medial to the substantia nigra. As the SCP ascended to the midbrain it coursed inferior then medial to the red nucleus and terminated and at midbrain structures (Fig. 8). Then, the SCP fibers made a sharp turn and formed the decussation of SCP and continued its course towards the diencephalon (Fig. 8). Some of the decussating fibers terminated at the opposite anterior hypothalamic area (Fig. 8). Some SCP 


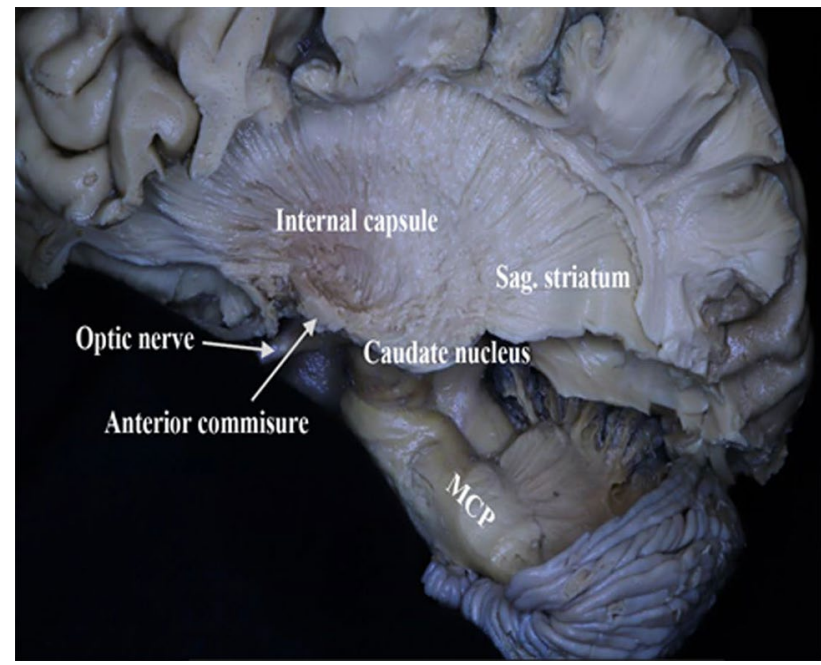

Fig. 5 The posterior and the retrolenticular fibers of the internal capsule MCP was exposed

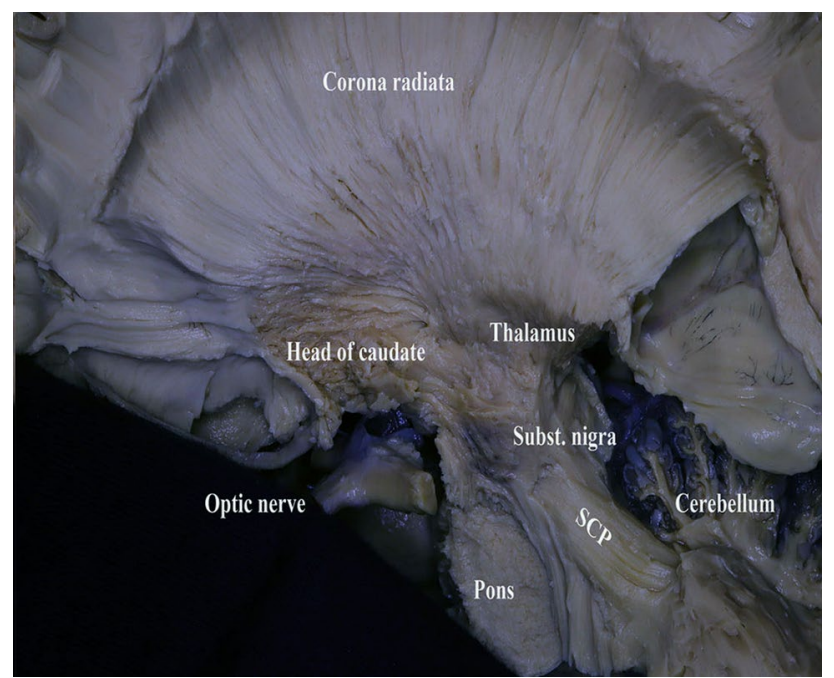

Fig. 6 The MCP was removed and the SCP and midbrain structures were exposed

fibers coursed inferolateral to the red nucleus and these uncrossed fibers reached the posterior hypothalamic area (Fig. 9). Further, we made a coronal cut from the right hemisphere at the level of splenium of the corpus callosum and the temporal horn of the lateral ventricle. We continued the dissection to expose the right SCP and left SCP (Fig. 10).

\section{DTI or tractography results}

We evaluated seven cases using high-resolution 3D diffusion tensor tractography (DTI) images. In five cases we have demonstrated unilateral left cerebello-hypothalamic fibers

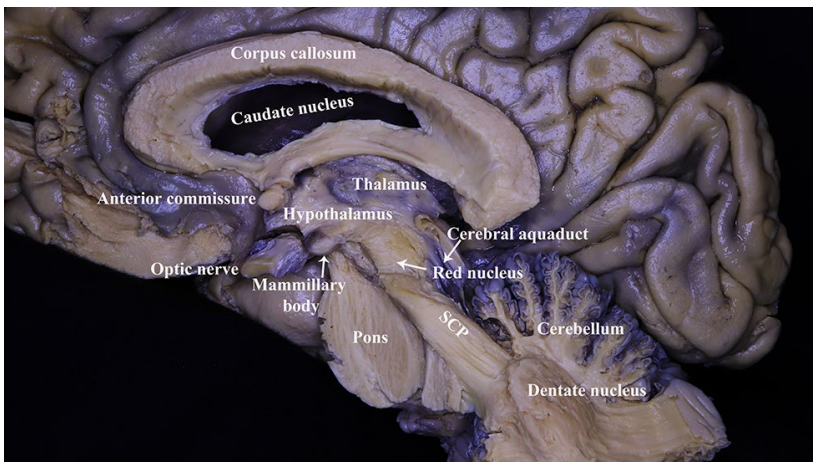

Fig. 7 The corona radiata has been removed and the opposite hemisphere was revealed. The corpus callosum was observed. The septum pellucidum was removed and deep to it was the opposite caudate nucleus. The interthalamic adesion, thalamus, hypothalamus were observed. Further down the mammillary bodies and the anterior commissure dentate nucleus and the SCP of the same side were revealed

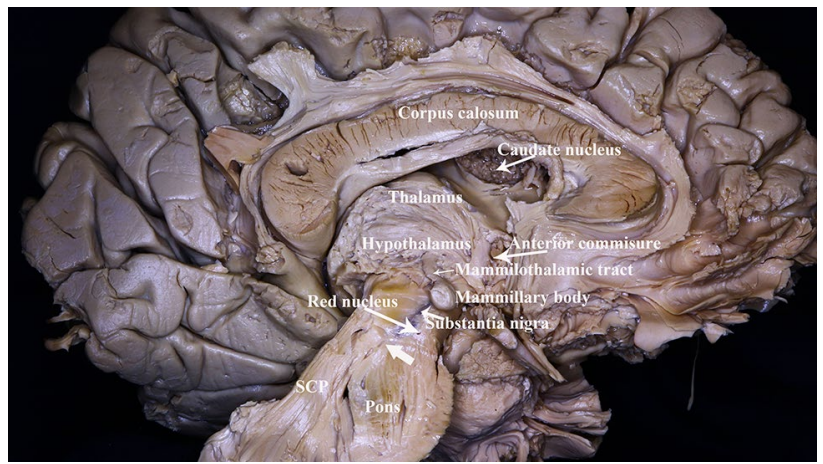

Fig. 8 Below the thalamus midbrain structures the substantia nigra and the red nucleus were revealed. The bundle of the SCP fibers inferior and medial to the red nucleus crossed to the opposite side forming the decussation of SCP and some terminated anterior hypothalamus (see white arrow)

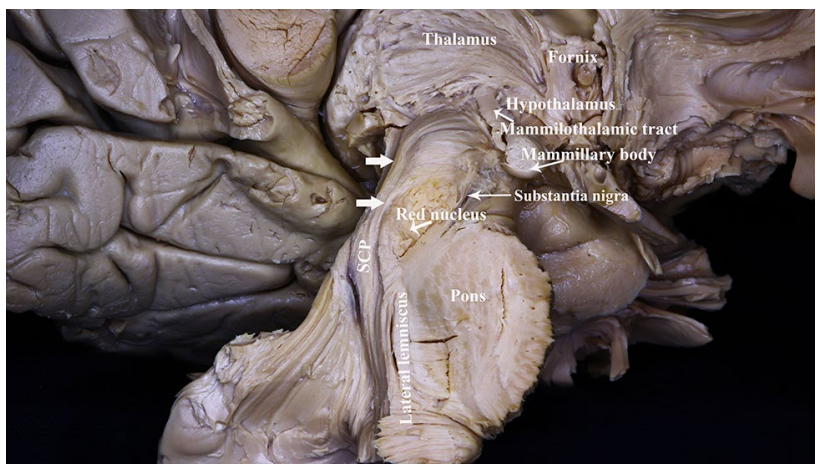

Fig. 9 Some of the SCP fibers passes inferior and lateral to the red nucleus ascended to the diencephalon and some fibers terminated at the posterior hypothalamic region (see white arrow) 


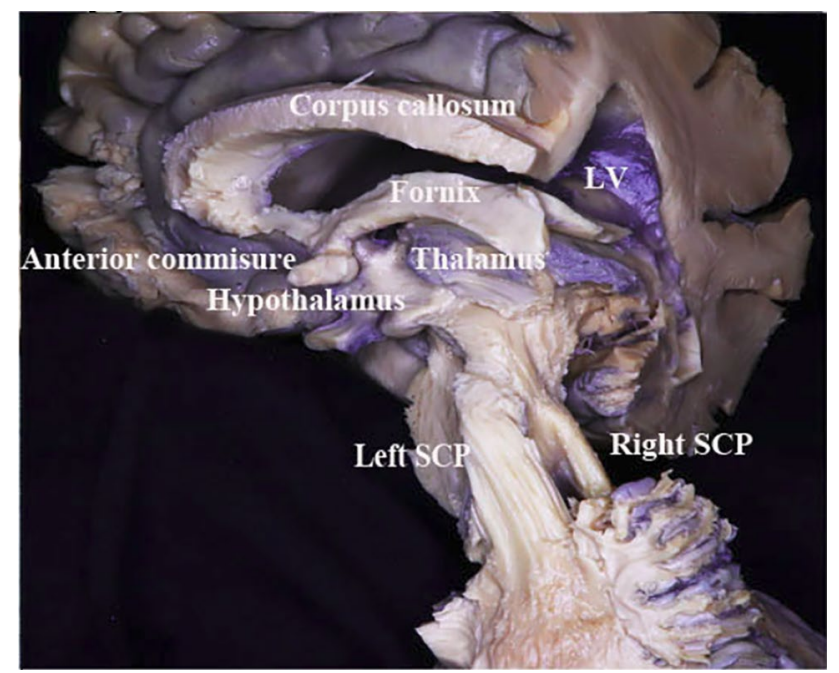

Fig. 10 The ascending fibers of the right and the left SCP

and in two cases bilateral cerebello-hypothalamic fibers. The DTI analyses of both the unilateral and bilateral cerebellohypothalamic tracts originated from the deep nuclei of cerebellum. These fibers reaching the hypothalamus blended with the fibers of the SCP (Fig. 11). Via the SCP they reached the ipsilateral medial-posterior hypothalamus in all cases. In two cases, these tracts extended to the ipsilateral internal capsules and terminated in the ipsilateral white matter of various brain lobes (Fig. 11).

\section{Discussion}

The present study, using both fiber dissection and DTI in adult human brains showed direct cerebello-hypothalamic connections. Both techniques showed direct cerebellohypothalamic fibers were localized within the SCP. The SCP fibers ascended to the level of midbrain reaching the red nucleus. The majority of the fibers terminated at the red nucleus and nearby structures in the midbrain, a bundle of fibers decussated inferior and medial to the red nucleus and reached the contralateral anterior hypothalamus and some fibers passed lateral to the red nucleus and reached the ipsilateral posterior hypothalamus (Fig. 12). The DTI results clearly showed the presence of direct ipsilateral cerebellohypothalamic fibers however, contralateral cerebello-hypothalamic could not be shown due limitations of the DTI and tractography technique (Nimsky et al. 2016).

Early evidence for cerebello-hypothalamic connection was presented by Wallenberg (1905). He described degeneration axons in the rabbit hypothalamus after a brainstem lesion which included the SCP. Jacobs (1965), reported degeneration in the hypothalamus following lesion of the SCP and cerebellar nuclei. Martin et al. (1974) described

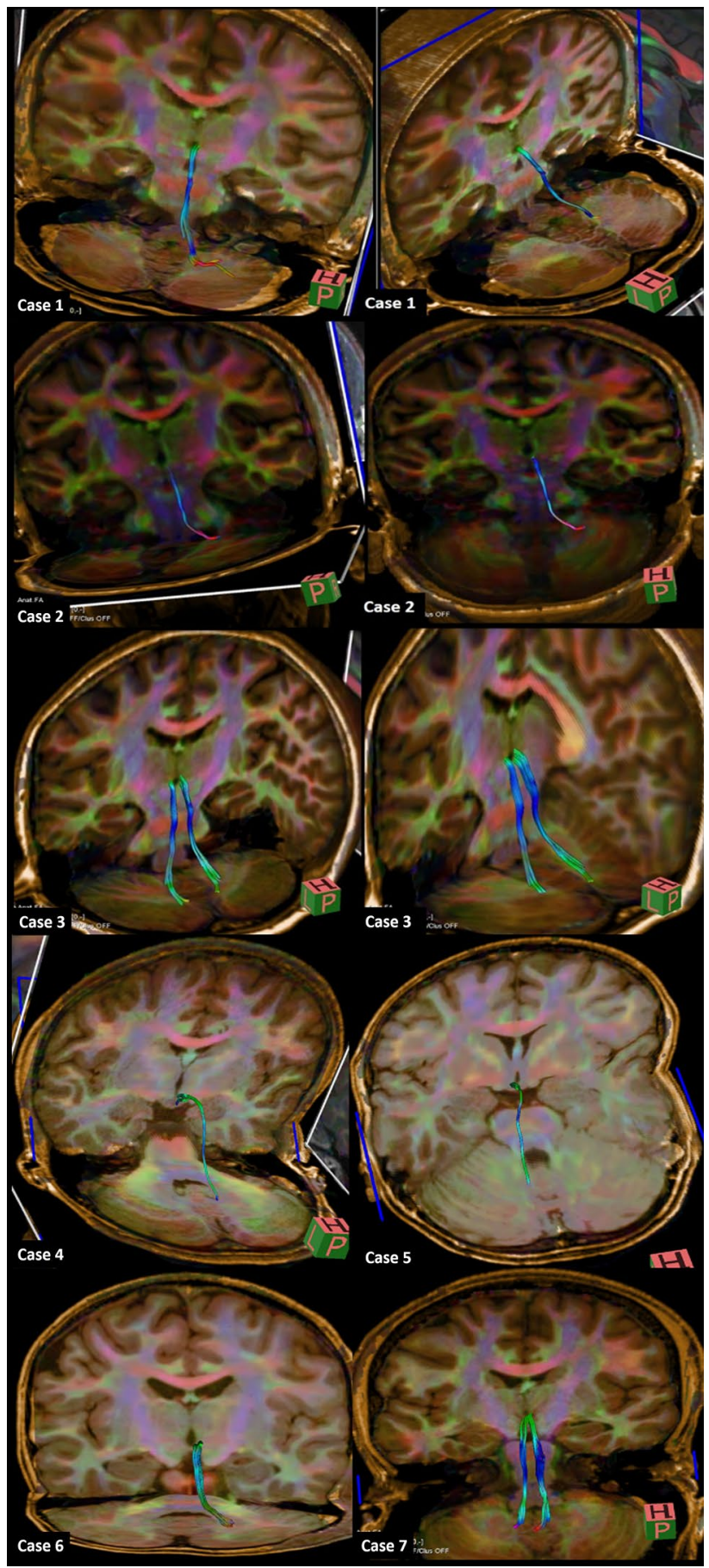

Fig. 11 The DTI images of seven cases showing the direct cerebellohypothalamic pathways via SCP. In five cases unilateral (case 1, 2, $4,5,6)$ and in two cases bilateral (case 3, 7) cerebello-hypothalamic pathways were traced

degeneration in the lateral hypothalamic area following the destruction of the medial cerebellar nucleus. Furthermore, experimental studies showed relations of the cerebellum with hypothalamic functions. Wen et al. (2004) reported that cerebellum participate in the cardiovascular regulation 


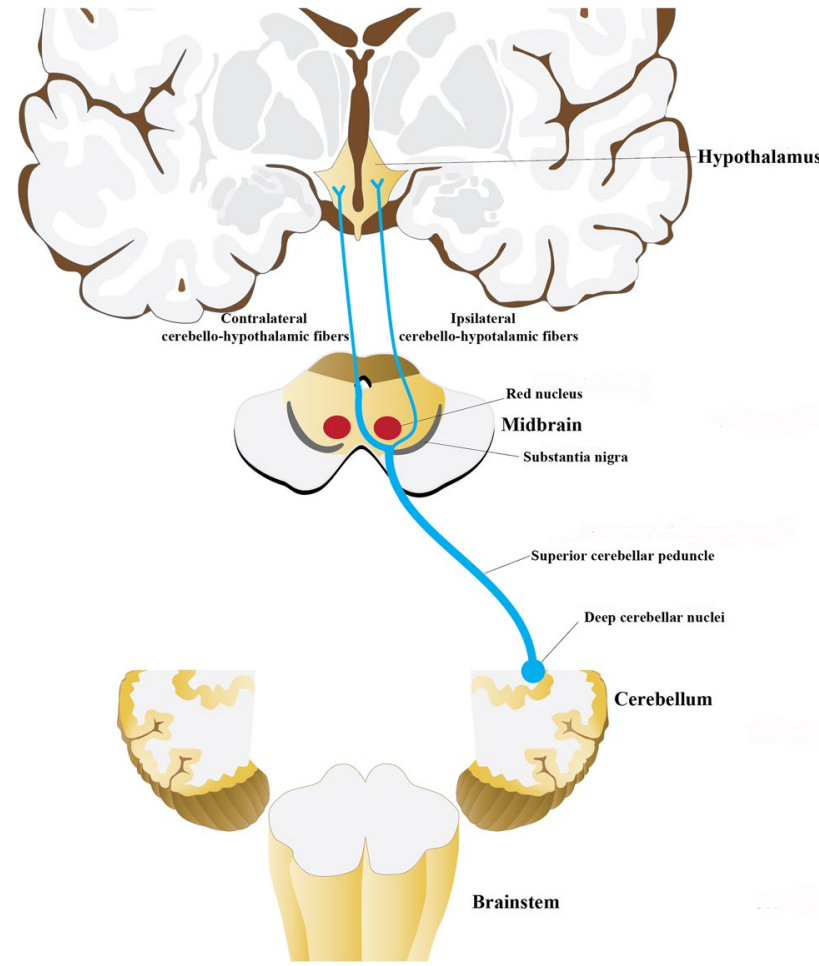

Fig. 12 The schematic illustration summarizes the ipsilateral and the contralateral cerebello-hypothalamic fibers within the SCP

and osmoregulation via the cerebello-hypothalamic projections. Li et al. (2017) demonstrated that electrical stimulation of cerebellar nuclei may elicit an excitatory, inhibitory or biphasic response of ventromedial hypothalamic nucleus of the hypothalamus, which is believed to be involved in feeding behavior, energy balance, and weight control. The stimulation of the dentate nucleus and SCP of patients with tremor experienced an unpleasant sensation of fear (Nashold and Slaughter 1969). Further, recent studies showed the role of cerebellar nuclei in the regulation of immune system $(\mathrm{Lu}$ et al. 2012; Peng et al. 2005, 2006; Cao et al. 2013). The results of neurological studies showed evidence of abnormal cerebellar processing can lead to alterations in mental functions. Studies reported a high rate of psychiatric and cognitive disorders in patients with degenerative cerebellar diseases (Leroi et al. 2002; Kish et al. 1994; D’Angelo and Casali 2013). A DTI study showed reduced fractional anisotropy in the white matter of the parietal association cortex and the left SCP in the early onset of schizophrenia patients (Kyriakopoulos et al. 2008). It is interesting that in the DTI of all 7 cases examined in the present study we were able to demonstrate the left cerebello-thalamic fibers but not the right. The left cerebello-thalamic fibers can be more dominant than right.

Experimental studies have described both direct (Cavdar et al. 2001; Haines et al. 1990) and indirect (Dietrichs et al.
1985; Wen et al. 2004) cerebello-hypothalamic connections. Dietrichs et al. (1985) described an indirect multisynaptic connections via lateral reticular nucleus in the cat. Other brain stem relay nuclei mediating hypothalamic input to cerebellum were described from the dorsal motor vagal nucleus, the periaqueductal grey, the nucleus of the solitary tract, the nucleus locus coeruleus and the raphe nuclei (Zheng et al. 1982; Dietrichs and Haines 1985; Dietrichs 1983; Somana and Walberg 1979). A recent DTI study showed the trajectory of an indirect cerebello-ponto-hypothalamic pathways (Kamali et al. 2018). Kamali et al. (2018) described cerebellar fibers reach the reticular formation of the pons through the middle cerebellar peduncles and then cross the midline within the reticular formations of the pons and project into the contralateral hypothalamic nuclei. The present study demonstrated the trajectory of the direct cerebello-hypothalamic connections via SCP using DTI and fiber dissection technique. Thus, the direct connections can be via SCP and the indirect connections can be via the MCP. The direct and indirect cerebello-hypothalamic connections may have fundamentally different functional properties compared to monosynaptic pathways.

An early study described ascending and descending limbs of the SCP. The ascending limb terminates at the red nucleus and other mid-brain structures and continues to terminate in the subthalamic area and thalamus. Rostral to the decussation the descending limb terminates pons, medulla oblongata and spinal cord (Martin et al. 1974; Asanuma et al. 1980; Faull and Carman 1978). Furthermore, Cajal (1972) reported that decussating fibers are collaterals of the ascending fibers. Bentivoglio and Kuypers (1982) used double retrograde tracer technique and showed that the vast majority of the neurons in the deep cerebellar nuclei distribute fibers to the contralateral diencephalon, probably mainly to the thalamus. They do not mention about the hypothalamic termination. The present fiber dissection and DTI technique show direct connections of the cerebellum with the hypothalamus. However, the two technique does not show whether these fibers are cerebello-hypothalamic or hypothalalamo-cerebellar pathways.

The DTI is a promising technique, however, the process has many limitations and pitfalls from acquiring the DTI scan to the tractography algorithm chosen and interpretation of the results. There are many versions, algorithm, and/or software's for DTI or tractography techniques (e.g. deterministic/probabilistic tractography, High Angular Resolution Diffusion Imaging, Q-Ball imaging, diffusion spectrum MRI tractography) (Lilja and Nilsson 2015). Each contains its own advantages or limitations. Further, tractography of brain stem and cerebellar structures is still a huge challenge for radiologists (Algin and Ozmen 2015). In the present study due to the above limitations of DTI and the low number cases we were unable to show the contralateral 
cerebello-hypothalamic fibers. Studies using 7 T MR with a larger series is necessary to define these fine tracts.

Being aware of the direct cerebello-hypothalamic fibers coursing within the SCP is of importance to the neurosurgeons both for preoperative counseling surgery (metastases invading the SCP and the dentate nucleus, gliomas of the tectum and tegmentum and brain stem lesions including diffused glioma and cavernomas) and to reduce the frequency of cerebello-hypothalamic defects. In addition, it can be used to interpret the unexplained deficit that may occur during regional surgery.

\section{Conclusion}

The present study demonstrates the direct cerebello-hypothalamic connections for the first time using fiber dissection and DTI technique in the human brain. Special care must be taken during the surgery of the region, furthermore, the knowledge of these connections can be a guide in the interpretation of radiological and neurological abnormalities involving SCP.

Acknowledgements The authors thank Professor Hakan Örer for the descriptive illustration which made the manuscript understandable.

\section{Compliance with ethical standards}

Conflict of interest None of the authors has any conflict of interest to disclose.

Ethical approval All cadavers used in this study was donated for medical student's dissections and research purposes. All procedures performed in studies were approved by the Institutional Ethics Committee of Koç University.

\section{References}

Algin O, Ozmen E (2015) Ectopic anterior cerebellum (ala lobule centralis). Neuroradiol J 28(3):278-280

Anderson G, Armstrong DM, Edgley SA (1987) Impulse activities of cerebellar neurons during locomotion in the cat. In: King JS (ed) New concepts in cerebellar neurobiology. Alan R. Liss, New York, pp 349-370

Asanuma C, Thach WT, Jones EG (1980) Nucleus interpositus projection to the spinal interneurons in monkey. Brain Res 24:49-68

Ban T, Inoue K, Ozaki S et al (1956) Interrelation between anterior lobe of cerebellum and hypothalamus in rabbit. Med J Osaka Univ 7:101-115

Bentivoglio M, Kuypers HG (1982) Divergent axon collaterals from rat cerebellar nuclei to diencephalon, mesencephalon, medulla oblongata and cervical cord. A fluorescent double retrograde labeling study. Exp Brain Res 46(3):339-356

Cajal SR (1972) Histologie du système nerveux. Instituto Ramony Cajal, Madrid
Cao BB, Huang Y, Lu JH et al (2013) Cerebellar fastigial nuclear GABAergic projections to the hypothalamus modulate. Immune function. Brain Behav Immun 27(1):80

Cavdar S, San T, Aker R (2001) Cerebellar connections to the dorsomedial and posterior nuclei of the hypothalamus in the rat. $\mathrm{J}$ Anat 198(Pt 1):37-45

Çavdar S, Özgür M, Kuvvet Y et al (2018a) Cortical, subcortical and brain stem connections of the cerebellum via the superior and middle cerebellar peduncle in the rat. J Integr Neurosci 17(3-4):609-618

Çavdar S, Özgur M, Kuvvet Y et al (2018b) The cerebello-hypothalamic and hypothalamo-cerebellar pathways via superior and middle cerebellar peduncle in the rat. Cerebellum 17(5):517-524

Chida K, Iadecola C, Underwood MD (1986) A novel vasodepressor response elicited from the rat cerebellar fastigial nucleus: the fastigial depressor response. Brain Res 370:378-382

Cohen D, Yarom Y (2000) Cerebellar on-beam and lateral inhibition: two functionally distinct circuits. J Neurophysiol 83(4):1932-1940

D'Angelo E, Casali S (2013) Seeking a unified framework for cerebellar function and dysfunction: from circuit operations to cognition. Front Neural Circuits 6:116

Dietrichs E (1983) Cerebellar cortical afferents from the periaqueductal grey in the cat. Neurosci Lett 41:21-26

Dietrichs E, Zheng ZH (1984) Are hypothalamo-cerebellar fibers collaterals from the hypothalamo-spinal projection? Brain Res 296(2):225-231

Dietrichs E, Haines DE, Qvist H (1985) Indirect hypothalamo-cerebellar pathway? Demonstration of hypothalamic efferents to the lateral reticular nucleus. Exp Brain Res 60:483-491

Faull RLM, Carman JB (1978) The cerebellofugal projections in the brachium conjunctivum of the rat. I. The contralateral ascending pathway. J Comp Neurol 178:495-518

Haines DE, Dietrichs E (1984) An HRP study of hypothalamo-cerebellar and cerebellohypothalamic connections in squirrel monkey (Saimiri sciureus). J Comp Neurol 229:559-575

Haines DE, Manto MU (2007) Clinical symptoms of cerebellar disease and their interpretation. Cerebellum 6(4):360-374

Haines DE, Dietrichs E, Sowa TE (1984) Hypothalamo-cerebellar and cerebello-hypothalamic pathways: a review and hypothesis concerning cerebellar circuits which may influence autonomic centers affective behavior. Brain Behav Evol 24(4):98-220

Haines DE, May PJ, Dietrichs E (1990) Neuronal connections between the cerebellar nuclei and hypothalamus in Macaca fascicularis: cerebello-visceral circuits. J Comp Neurol 299(1):106-122

Haines DE, Dietrichs E, Mihailoff GA, McDonald EF (1997) The cerebellar-hypothalamic axis: basic circuits and clinical observations. Int Rev Neurobiol 41:83-107

Jacobs VL (1965) The cerebellofugal system in the tarsius (Tarsiidae carbonarius) and the marmoset (Oedipomidas oedipus). In: Doctoral dissertation. Lawrence, Kansas, USA: University of Kansas

Kamali A, Karbasian N, Rabiei P et al (2018) Revealing the cerebelloponto-hypothalamic pathway in the human brain. Neurosci Lett 677:1-5

Kish SJ, El-Awar M, Stuss D et al (1994) Neuropsychological test performance in patients with dominantly inherited spinocerebellar ataxia: relationship to ataxia severity. Neurology 44:1738-1746

Klingler J, Ludwig E (1956) Atlas cerebrihumani. Karger, Basel

Kyriakopoulos M, Vyasa NS, Barkerb GJ et al (2008) A diffusion tensor imaging study of white matter in early-onset schizophrenia. Biol Psychiatry 63:519-523

Leroi I, O'Hearn E, Marsh L (2002) Psychopathology in patients with degenerative cerebellar diseases: a comparison to Huntington's disease. Am J Psychiatry 159(8):1306-1314

Li B, Zhuang QX, Gao HR, Wang JJ, Zhu JN (2017) Medial cerebellar nucleus projects to feeding-related neurons in the ventromedial hypothalamic nucleus in rats. Brain Struct Funct 222(2):957-971 
Lilja Y, Nilsson DT (2015) Strengths and limitations of tractography methods to identify the optic radiation for epilepsy surgery. Quant Imaging Med Surg 5(2):288-299

Lu JH, MaoHN Cao BB et al (2012) Effect of cerebellohypothalamic glutamatergic projections on immune function. Cerebellum 11(4):905-916

Martin GF, King JS, Dom R (1974) The projections of the deep cerebellar nuclei of the opossum, Didelphis marsupialis virginiana. J fuXr Hirnforschung 15:545-573

Middleton FA, Strick PL (1997) Dentate output channels: motor and cognitive components. Progress in Brain Res 114:553-566

Middleton FA, Strick PL (1998) Cerebellar output: motor and cognitive channels. Trends in Cognitive Sci 2:348-354

Nashold BS Jr, Slaughter DG (1969) Effects of stimulating or destroying the deep cerebellar regions in man. J Neurosurg 31(2):172-186

Nimsky C, Bauer M, Carl B et al (2016) Merits and limits of tractography techniques for the uninitiated. Adv Tech Stand Neurosurg 43:37-60

Peng YP, Qiu YH, Cao BB et al (2005) Effect of lesions of cerebellar fastigial nuclei on lymphocyte functions of rats. Neurosci Res $51: 275-284$

Peng YP, Qiu YH, Qiu J (2006) Cerebellar interposed nucleus lesions suppress lymphocyte function in rats. Brain Res Bull 71:10-17
Somana E, Walberg F (1979) Cerebellar afferents from the nucleus of the solitary tract. Neurosci Lett 11:41-47

Wallenberg A (1905) Sekundäre Bahnen aus dem frontalen sensiblen Trigeminuskerne des Kaninchens. Anat Anz 26:145-155

Wayner MJ, Barone FC, Loullis CC (1991) The lateral hypothalamus and adjunctive behavior. In: Morgane PJ, Panksepp J (eds) Handbook of the hypothalamus, vol 3. Part B: behavioral studies of the hypothalamus. Marcel Dekker, New York, pp 107-146

Wen YQ, Zhu JN, Zhang YP et al (2004) Cerebellar interpositus nuclear inputs impinge on paraventricular neurons of the hypothalamus in rats. Neurosci Lett 370(1):25-29

Zanchetti A, Zoccolini A (1954) Autonomic hypothalamic outbursts elicited by cerebellar stimulation. J Neurophysiol 17(5):475-483

Zheng ZH, Dietrichs E, Walberg F (1982) Cerebellar afferent fibres from the dorsalmotor vagal nucleus in the cat. Neurosci Lett 32(2):113-118

Publisher's Note Springer Nature remains neutral with regard to jurisdictional claims in published maps and institutional affiliations. 